\title{
Innovatives Brustkrebs-Forschungsprojekt HYPMED der Uniklinik RWTH Aachen erhält EU-Fördermittel in Höhe von 6 Millionen $€$
}

Gutachter loben ausdrücklich das wissenschaftliche und klinische Potenzial des Projektvorschlags.

Aachen/ Brüssel, 07.09.2015 - Der federführend von der Uniklinik RWTH Aachen aufgestellte Projektvorschlag HYPMED (Entwicklung eines hybriden MRT/PETSystems für die Diagnose von Brustkrebs) erhielt von den Gutachtern des EU-Programms „Horizon 2020“ Bestnoten und eine uneingeschränkte Förderungsempfehlung. Höchstes Lob fand die Gutachterkommission für das innovative Konzept, das mit diesem Antrag verfolgt wird: „Das Projekt besitzt ein extrem hohes Potenzial. Wir rechnen damit, dass sich der im HYPMED-Projekt verfolgte neuartige Ansatz durchsetzen und mittels zielgerichteter MR/ PET-Bildgebung eine frühere Diagnose von Brustkrebs ermöglichen wird.“

„Das HYPMED-Projekt verbindet auf ideale Weise medizinische Kompetenz mit physikalisch-technischer und ingenieurwissenschaftlicher Expertise. Es zeigt exemplarisch, welches Potenzial gerade die Uniklinik RWTH Aachen auf dem Sektor Medizin und Technik bietet", unterstreicht die Leiterin des Projekts, Univ.-Prof. Dr. med. Christiane Kuhl, Direktorin der Klinik für Diagnostische und Interventionelle Radiologie an der Uniklinik RWTH Aachen. Gemeinsam mit Univ.-Prof. Dr.-Ing. 
Volkmar Schulz, Leiter des Lehr- und Forschungsgebietes Physik der Molekularen Bildgebungssysteme am Institut für Experimentelle Molekulare Bildgebung und Mitglied des Fachbereiches Physik der RWTH Aachen, wird sie mit verschiedenen Partnern aus dem In- und Ausland am HYPMED-Projekt arbeiten. „Das Horizon 2020-Programm ist hochkompetitiv; gerade einmal $2 \%$ der eingereichten Projekte werden gefördert“, erklärt Prof. Schulz. „Aber besonders außergewöhnlich ist es, dass ein Projekt von den Gutachtern in sämtlichen Bereichen mit maximaler Punktzahl bewertet wird.“

\section{Brustkrebs frühzeitig diagnostizie- ren und gezielt behandeln \\ $\nabla$}

Brustkrebs ist immer noch die häufigste Krebstodesursache von Frauen, weshalb die Suche nach neuen Möglichkeiten, die Krankheit früh zu diagnostizieren und gezielt zu behandeln, unverändert im Fokus der wissenschaftlichen Forschung steht. Das HYPMED-Projekt verfolgt die Entwicklung eines neuartigen Geräts für die medizinische Bildgebung - von den physikalischen Grundlagen über die ingenieurwissenschaftliche Konstruktion bis zur klinischen Erprobung. Das neue Untersuchungsgerät wird eine bislang unerreichte Kombination aus Brust-MRT und PET (Positronen-Emissions-Tomografie) ermöglichen und damit weltweit den Stand der Technik auf diesem Gebiet neu definieren. „Mit unserem Ansatz wird es möglich sein, jedes beliebige MR-System bei Bedarf in ein sogenanntes ,Hybridsystem` zu verwandeln, mit dem wir auch kleinste Veränderungen erkennen oder die biologische Aggressivität von Tumoren besser einschätzen können“, erläutert Prof. Kuhl. „Solche Bildgebungsverfahren werden für die zielgerichtete Therapie dringend benötigt.“ Dies trifft nicht nur auf Brustkrebs zu. Vielmehr wird das neue Konzept auch für andere Erkrankungen bislang ungeahnte Diagnosemöglichkeiten eröffnen. „Mit dem Erfolg des HYPMED-Projekts werden wir ein ganz neues Kapitel in der Medizinischen Bildgebung aufschlagen“, so Prof. Kuhl.

\section{Kontakt:}

Prof. Dr. Christiane Kuhl

Direktorin der Klinik für Diagnostische

und Interventionelle Radiologie der

Uniklinik der RWTH Aachen

ckuhl@ukaachen.de 